\title{
Modelo de aprovechamiento sustentable de residuos sólidos orgánicos en Cundinamarca, Colombia
}

\author{
Model of sustainable utilization of organic solids waste in Cundinamarca, Colombia
}

Solanyi Castañeda-Torres ${ }^{*}$, Juan Pablo Rodríguez-Miranda²

1 Universidad Distrital Francisco José de Caldas. Bogotá, Colombia

2 Grupo de investigación AQUAFORMAT, Universidad Distrital Francisco José de Caldas. Bogotá, Colombia

Fecha de recepción: Julio 29 - $2016 \quad$ Fecha de revisión: Diciembre 5 - $2016 \quad$ Fecha de aceptación: Marzo 24 - 2017

Castañeda-Torres S, Rodríguez-Miranda JP. Modelo de aprovechamiento sustentable de residuos sólidos orgánicos en Cundinamarca, Colombia. Univ. Salud. 2017;19(1):116-125. DOI: http://dx.doi.org/10.22267/rus.171901.75

\begin{abstract}
Resumen
Introducción. Este artículo considera una propuesta de un modelo de aprovechamiento de residuos sólidos orgánicos para el departamento de Cundinamarca, el cual responde a la necesidad de una herramienta que apoye en la toma de decisiones para la planificación y gestión de los residuos sólidos orgánicos. Objetivo. Realizar una aproximación de un modelo de optimización conceptual, técnico y matemático para el apoyo en la toma de decisiones con el fin de minimizar impactos ambientales. Materiales y métodos. El tipo de investigación aplicada en este trabajo se considera descriptiva, dado que se presentan algunas características fundamentales del fenómeno homogéneo estudiado y se considera adicionalmente cuasi experimental. El cálculo del modelo para plantas del departamento está basado en tres ejes (ambiental, económico y social), presentes en la ecuación general de optimización. Resultados. Se obtiene un modelo de aprovechamiento de residuos sólidos orgánicos en las técnicas de tratamiento biológico de compostaje aeróbico y lombricultivo, optimizando el sistema con el ahorro de emisiones de gases efecto invernadero emitidos a la atmósfera, y en la reducción total del costo de disposición final de residuos sólidos orgánicos en relleno sanitario. Basándose en el principio económico de utilidad que determina la viabilidad ambiental y sostenibilidad en las plantas de aprovechamiento de residuos sólidos orgánicos para el departamento, los abonos orgánicos como el compost y humus capturan carbono e nitrógeno que reducen las toneladas de $\mathrm{CO}_{2}$.
\end{abstract}

Palabras clave: Residuo sólido; compostaje; lombriculura. (Fuente: DeCS, Bireme).

\begin{abstract}
Introduction: This article considers a proposal of a model of use of organic solids waste for the department of Cundinamarca, which responds to the need for a tool to support decision-making for the planning and management of organic solids waste. Objective: To perform an approximation of a conceptual technical and mathematician optimization model to support decisionmaking in order to minimize environmental impacts. Materials and methods: A descriptive study was applied due to the fact that some fundamental characteristics of the studied homogeneous phenomenon are presented and it is also considered to be quasi experimental. The calculation of the model for plants of the department is based on three axes (environmental, economic and social), that are present in the general equation of optimization. Results: A model of harnessing organic solids waste in the techniques of biological treatment of composting aerobic and worm cultivation is obtained, optimizing the system with the emissions savings of greenhouse gases spread into the atmosphere, and in the reduction of the overall cost of final disposal of organic solids waste in sanitary landfill. Based on the economic principle of utility that determines the environmental feasibility and sustainability in the plants of harnessing organic solids waste to the department, organic fertilizers such as compost and humus capture carbon and nitrogen that reduce the tons of $\mathrm{CO}_{2}$.
\end{abstract}

Keywords: Solid organic waste; optimization model; composting; vermiculture. (Source: DeCS, Bireme).

\footnotetext{
*Autor de correspondencia

Juan Pablo Rodríguez-Miranda

e-mail: sct.sustentable@gmail.com
} 


\section{Introducción}

Se estima que el crecimiento poblacional hacia las ciudades y municipios ha aumentado la tasa de eliminación de residuos orgánicos, provocando la apertura de nuevos vertederos e incineradores ${ }^{1}$. Es por ello, que se hace necesaria la gestión sostenible de residuos municipales en las fases de impacto como son la planificación, el diseño, la operación y la clausura. En el espectro de tecnologías nuevas y existentes acerca de la gestión de residuos se han abarcado estrategias para el mantenimiento de la calidad del ambiente y sostenimiento de metas futuras. Este tipo de tecnologías permite que industrias y agencias gubernamentales, hagan frente a las necesidades comunes en el reciclaje de materiales biodegradables, con el fin de ampliar el suministro de energía renovable, y a su vez ofrecer opciones socialmente más aceptables para preservar la biodiversidad y los ecosistemas naturales ${ }^{2}$.

Dentro de la perspectiva de aumentar la energía renovable para mitigar el calentamiento global y en relación al desarrollo de alternativas de energía sostenible, la biomasa se ha considerado como una posible fuente de energía renovable. La configuración de un modelo permite optimizar el sistema con base en los objetivos económicos y ambientales. La energía es un bien esencial para el bienestar del hombre por ello el aumento en la producción y consumo de energía renovable y no renovable conduce a la investigación de nuevos medios de utilización y reutilización de nuevas fuentes de energía ${ }^{3}$.

Los modelos de optimización se enfocan principalmente en la gestión de residuos, incluyendo los sistemas de energía ${ }^{4}$. Los residuos son un recurso limitado y tiene diversas formas de tratamiento, entre estos se encuentra el reciclado y la utilización en la producción de energía ${ }^{4}$. Los modelos de optimización permiten mejorar los procesos y emitir parámetros que determinan una decisión mediante análisis matemáticos ${ }^{4}$, para optimizar las decisiones de inversión de energía endógena, y dar cumplimiento a un fin específico ${ }^{1}$. Las metodologías son implementadas por las empresas y/o municipios con el fin de obtener estrategias óptimas de inversión y para la planificación energética nacional analizando perspectivas del sistema energético ${ }^{1}$. El enfoque matemático que se le da en la mayoría de los casos es programación lineal, que permite maximizar o minimizar funciones específicas de las actividades en beneficio de la gestión de residuos, está sujeto a limitaciones operativas ${ }^{5}$.

En Colombia, especialmente en el Departamento de Cundinamarca el aprovechamiento de residuos sólidos orgánicos se ve como un proceso aislado dentro del servicio de aseo público ${ }^{6}$. El tratamiento de estos residuos se hace en vertederos o incineradoras que a menudo se encuentran en zonas minoritarias o deprimidas, generando un incremento en problemas de salud y de pobreza 7,8 . La producción de residuos sólidos orgánicos por cada habitante se encuentra alrededor de $0,7 \mathrm{~kg} / \mathrm{hab}$ día ${ }^{9-11}$. Actualmente, existen problemas en el sistema de aprovechamiento en los procesos de compostaje y lombricultivo del Departamento de Cundinamarca, presentando riesgos ambientales y de salud pública ${ }^{6}$. En la información suministrada en la resolución defensorial No 62 del Departamento de Cundinamarca, se evidencia la situación de las plantas de compostaje y lombricultivo del Departamento de Cundinamarca $^{12}$. Se realizaron visitas en las plantas de Guaduas y se encontró construcciones de composteras para aplicación de la técnica de lombricultivo; estas dificultan el volteo de los residuos, y por lo general se realiza en pilas ${ }^{6}$. El proceso de tamizaje se realiza manualmente, para proceder al empaque del producto, almacenamiento y comercialización. De este modo, la propuesta de aprovechamiento sustentable pretende aproximarse a un modelo de optimización conceptual, técnico y matemático para el apoyo en la toma de decisiones con el fin de minimizar impactos ambientales.

\section{Materiales y métodos}

El tipo de investigación aplicada al desarrollo del modelo de aprovechamiento sustentable de los residuos sólidos orgánico es descriptivo, por 
cuanto se detalla algunas características fundamentales del fenómeno homogéneo estudiado ${ }^{13}$; luego se registra información según va ocurriendo el fenómeno, en este caso, la obtención de los datos de las plantas de aprovechamiento de residuos sólidos orgánicos en el Departamento de Cundinamarca; sin embargo, según el análisis y alcance de los resultados la investigación también fue cuasi experimental ${ }^{14}$, debido a que existe una relación causal (causa - efecto), teniendo en cuenta que el manejo de los procesos se hace sin considerar algunos parámetros factores fisicoquímicos que afectan su calidad, como el contenido de humedad, la relación Carbono/Nitrógeno $(\mathrm{C} / \mathrm{N})$, la composición bioquímica y aspectos físicos como: el $\mathrm{pH}$, la temperatura y la textura del compostaje y lombricultivo ${ }^{6}$, que en condiciones de control riguroso de los factores pueden afectar el resultado del análisis.

El desarrollo de la metodología del modelo toma referentes de información primaria y secundaria, esenciales para visitar las plantas de aprovechamiento de residuos sólidos orgánicos en el Departamento de Cundinamarca, luego se realiza un análisis del mercado en términos del precio de venta y aspectos técnicos para el aprovechamiento de los residuos sólidos orgánicos (RSO). Posteriormente, con la información obtenida, se realizan cálculos de producción de RSO, se elabora el modelo conceptual, técnico y matemático y finalmente el software de cálculo.

Se establece cuatro fases metodológicas, la primera de recopilación de información diagnóstica, científica y técnica. En la segunda, se involucra parámetros que vinculan al desarrollo sostenible en sus tres dimensiones. En la tercera fase, se establece el cálculo proyectado de la población por municipio. Obteniendo el cálculo de ton/año de las provincias que conforman el departamento. Finalmente, se desarrolla el modelo conceptual, técnico y matemático para el desarrollo de software (Figura 1).

Figura 1. Fases en metodología de la investigación

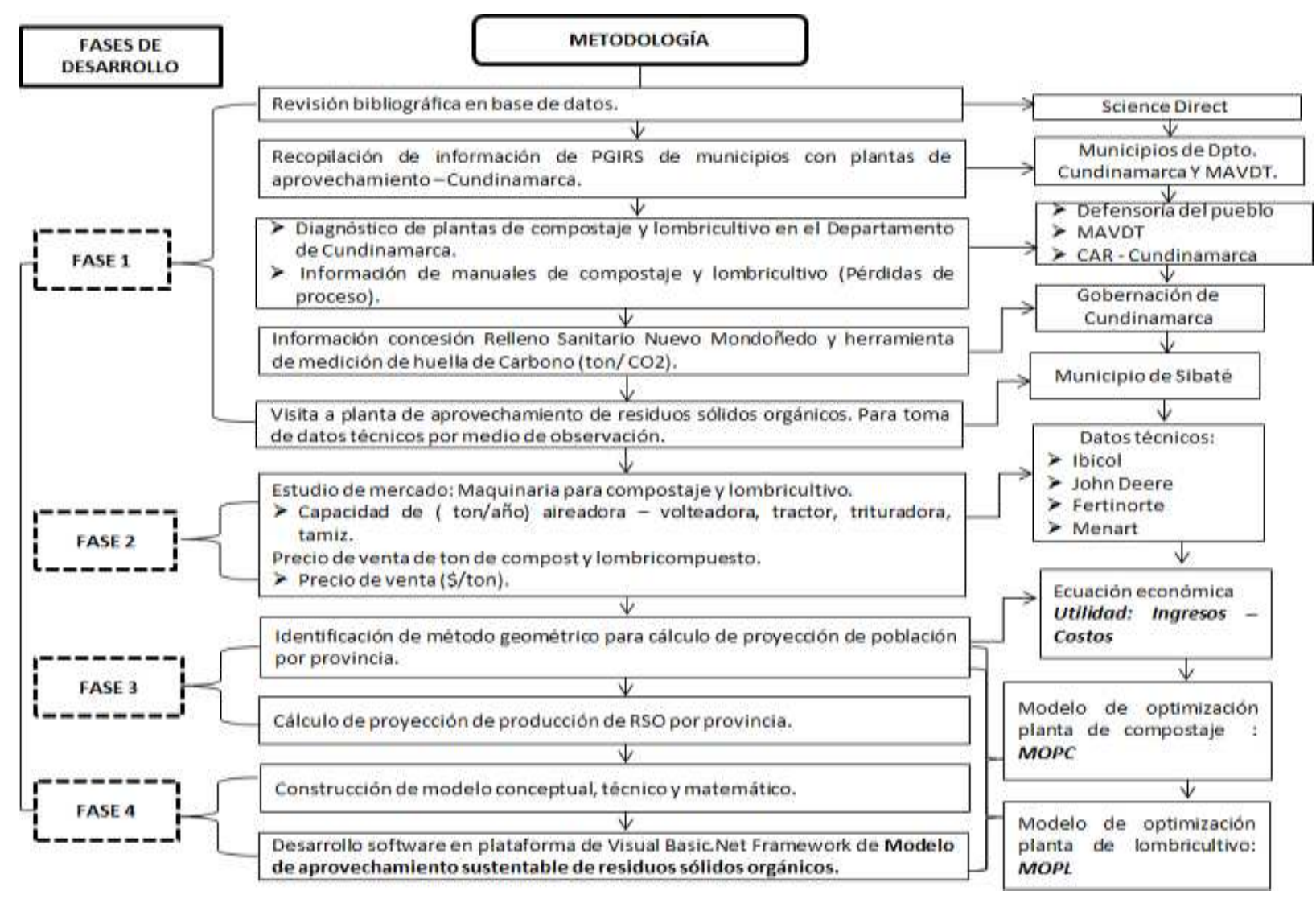




\section{Resultados}

\section{Cálculo de emisiones toneladas de $\mathrm{CO}_{2}$ para el Departamento de Cundinamarca}

Se elaboró el cálculo de las toneladas equivalentes de $\mathrm{CO}_{2}$, a través de la herramienta de huella de carbono presentado por la gobernación de Cundinamarca ${ }^{15,16}$, para cada una de las provincias del Departamento en la generación de residuos sólidos orgánicos en los años 2010, 2020, 2030, 2040. (Tabla 1)17,18.

Tabla 1. Cálculo de emisiones de toneladas de $\mathrm{CO}_{2}$

\begin{tabular}{|c|c|c|c|c|c|c|c|c|}
\hline PROVINCIA & $\begin{array}{c}\text { Población/ } \\
2010 \\
\end{array}$ & $\begin{array}{c}\text { Población/ } \\
2020\end{array}$ & $\begin{array}{c}\text { Población/ } \\
2030 \\
\end{array}$ & $\begin{array}{c}\text { Población/ } \\
2040 \\
\end{array}$ & $\begin{array}{l}\text { Generación } \\
\text { anual/2010 }\end{array}$ & $\begin{array}{r}\text { Generación } \\
\text { anual/2020 }\end{array}$ & $\begin{array}{c}\text { Generación } \\
\text { anual/2030 }\end{array}$ & $\begin{array}{r}\text { Generación } \\
\text { anual/2040 }\end{array}$ \\
\hline Medina & 87.726 & 100.437 & 114.990 & 131.652 & 159 & 165 & 170 & 176 \\
\hline Magadalena Centro & 163.357 & 178.042 & 194.048 & 211.492 & 264 & 262 & 261 & 259 \\
\hline Bajo Magdalena & 68.701 & 79.579 & 92.179 & 106.775 & 623 & 722 & 836 & 969 \\
\hline Rionegro & 106.995 & 111.895 & 117.020 & 122.379 & 682 & 694 & 707 & 720 \\
\hline Oriente & 86.282 & 90.102 & 94.092 & 98.258 & 779 & 804 & 829 & 855 \\
\hline Guavio & 29.112 & 28.919 & 28.727 & 28.537 & 783 & 817 & 854 & 891 \\
\hline Almeidas & 17.553 & 18.152 & 18.771 & 19.412 & 796 & 911 & 1.043 & 1.194 \\
\hline Gualivá & 85.868 & 88.576 & 91.370 & 94.252 & 971 & 1.015 & 1.062 & 1.110 \\
\hline Ubaté & 75.149 & 76.528 & 77.932 & 79.361 & 1.061 & 1.162 & 1.274 & 1.396 \\
\hline Tequendama & 437.762 & 551.819 & 695.593 & 876.826 & 1.232 & 1.305 & 1.382 & 1.463 \\
\hline Alto Magdalena & 376.374 & 340.128 & 307.372 & 277.770 & 1.482 & 1.615 & 1.760 & 1.919 \\
\hline Sumapaz & 490.996 & 629.348 & 806.685 & 1.033 .992 & 1.852 & 2.153 & 2.502 & 2.908 \\
\hline Sabana Occidente & 204.185 & 237.315 & 275.821 & 320.574 & 3.414 & 3.086 & 2.788 & 2.520 \\
\hline Sabana Centro & 135.789 & 143.801 & 152.286 & 161.271 & 3.971 & 5.006 & 6.310 & 7.955 \\
\hline Soacha & 116.926 & 128.139 & 140.427 & 153.894 & 4.454 & 5.709 & 7.318 & 9.380 \\
\hline
\end{tabular}

Se estableció el ahorro de emisiones en toneladas de $\mathrm{CO}_{2}$ para cada intervalo de tiempo con el aumento de generación de residuos sólidos orgánicos y el procesamiento de los mismos en condiciones adecuadas de tecnología, disminución de emisiones de $\mathrm{CO}_{2}$ al ambiente. Los resultados evidencian que las técnicas de aprovechamiento como compostaje y lombricultivo aportan en la captura de carbono y nitrógeno, además en beneficios ambientales para la reparación de suelos, creación de empleos directos e indirectos, salud humana y beneficios económicos para la comunidad local. Con la construcción de las plantas habrá un ahorro de emisiones de toneladas equivalentes $/ \mathrm{CO}_{2}$ presentado en la (Figura 2).
Se comparó las utilidades en el tiempo desde el año 2010 al año 2040 con intervalo de diez años, y se evidenció que económicamente es más rentable la técnica de aprovechamiento compostaje en comparación con lombricultivo. Los ingresos de compost superan los ingresos de lombricompuesto, porque existe mayor eficiencia en la producción de toneladas generadas de compost y lombricompuesto respectivamente. En la Figura 3 se presenta la comparación de utilidades en el trascurso del 2010 al 2040 en el aprovechamiento de estos modelos. 


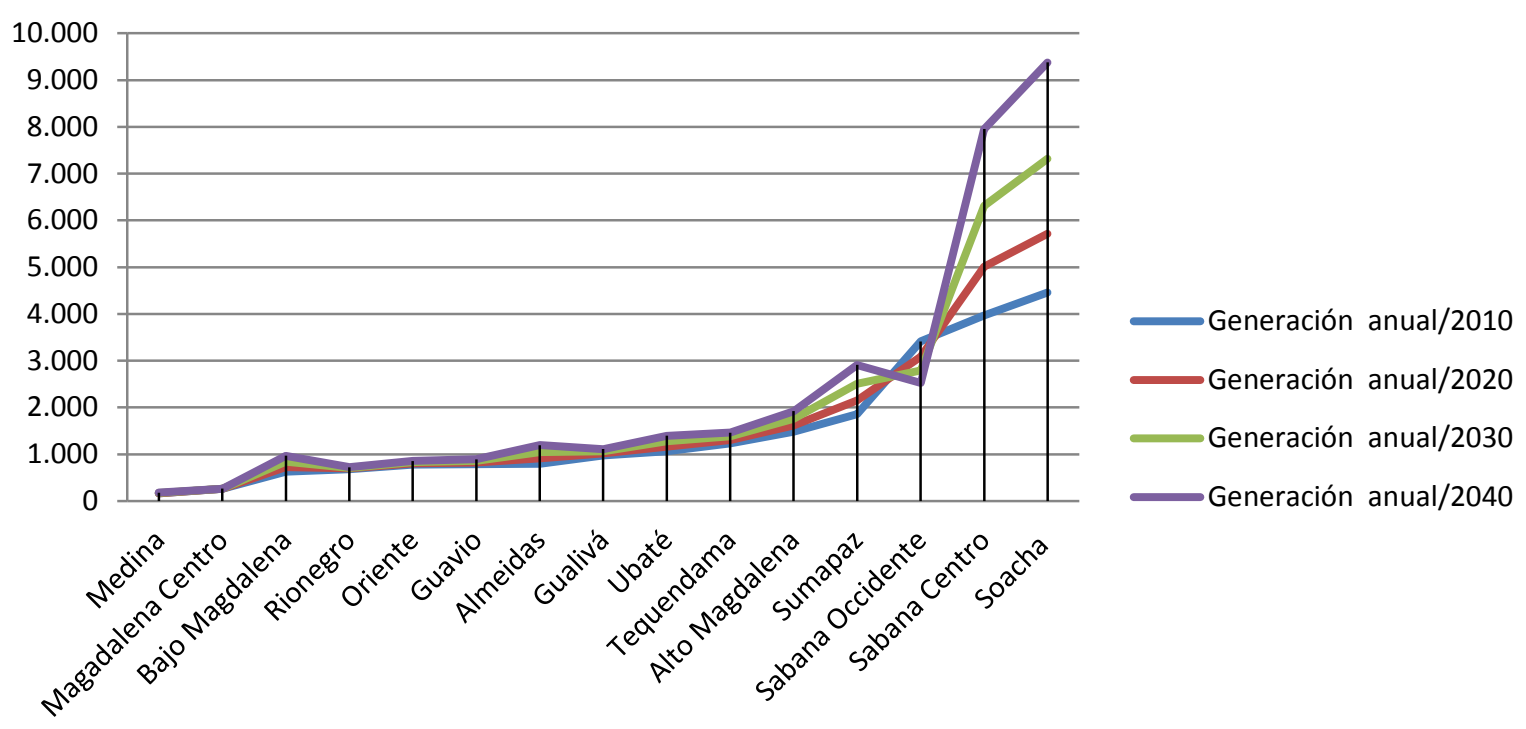

Provincia

Figura 2. Ahorro de emisiones de $\mathrm{CO}_{2}$ en compostaje y/o lombricultivo

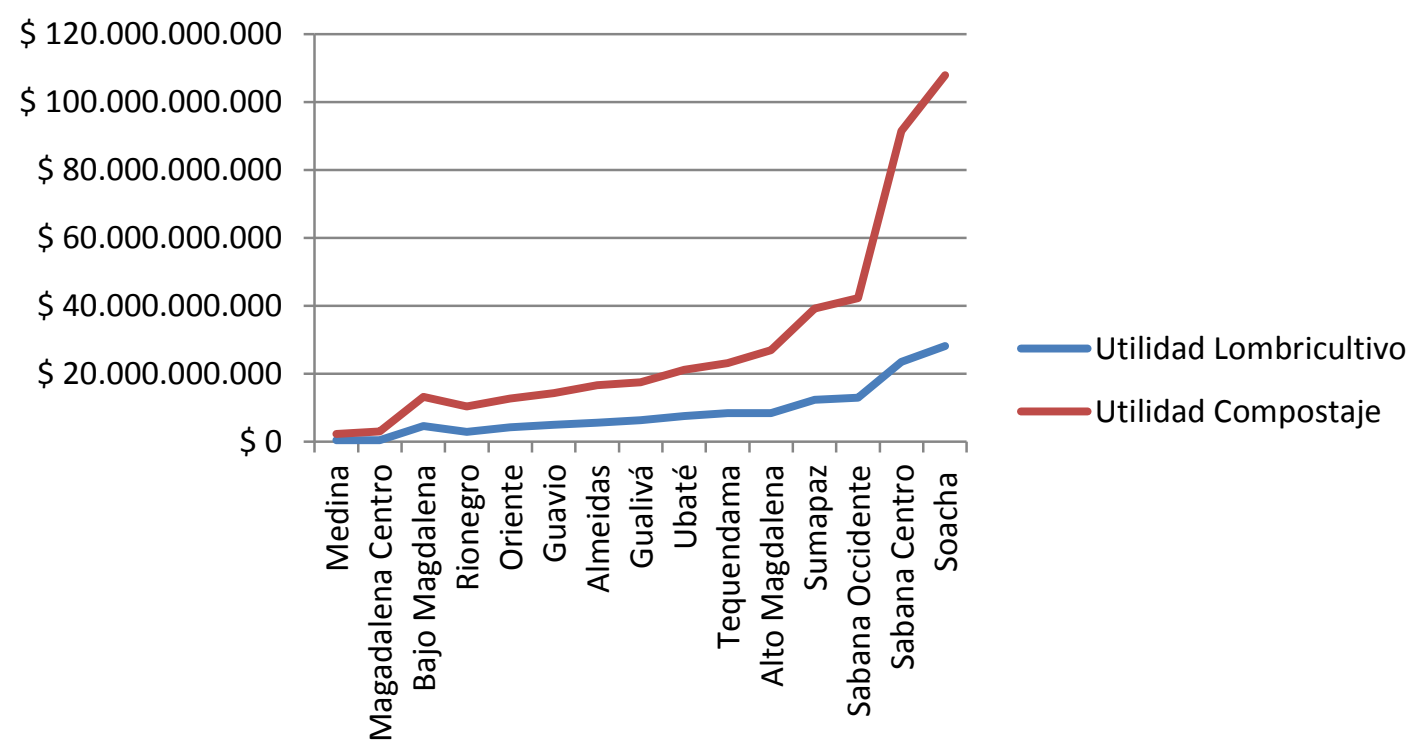

Figura 3. Comparación de utilidades de compostaje y lombricultivo 2010-2040

Modelo de aprovechamiento sustentable de residuos sólidos orgánicos en las técnicas de compostaje aeróbico y lombricultivo

Inicialmente para la construcción del modelo optimización de las plantas de compostaje y lombricultivo, se parte de la teoría de utilidad económica, expresada en la (Ecuación 1) ${ }^{18}$.

Ecuación 1. Ecuación de utilidad económica Utilidad $=$ Ingresos - Costos $^{19}$ 
El modelo de aprovechamiento sustentable de residuos sólidos orgánicos incluye los factores de cálculo económico, para el desarrollo del modelo de optimización de compostaje y lombricultivo, que se relacionan en la Tabla 2.

Tabla 2. Desglose de abreviaciones de ecuación de utilidad

\begin{tabular}{|c|c|c|}
\hline Abreviaciones & Tipo & Desglose \\
\hline I & Ingresos & $\begin{array}{l}\text { Precio de venta de } \\
\text { compost } y / o \\
\text { lombricultivo }\end{array}$ \\
\hline CI & $\begin{array}{l}\text { Costos de } \\
\text { Inversión }\end{array}$ & $\begin{array}{l}\text { Costos de } \\
\text { maquinaria + Costos } \\
\text { de terreno }\end{array}$ \\
\hline CD & $\begin{array}{l}\text { Costos de } \\
\text { Disposición }\end{array}$ & $\begin{array}{l}\text { Costo de cobro de } \\
\text { relleno sanitario por } \\
\text { disponer toneladas } \\
\text { de residuos sólidos } \\
\text { orgánicos. }\end{array}$ \\
\hline
\end{tabular}

De lo anterior, se desglosa la explicación de las variables que componen el modelo de aprovechamiento sustentable de los residuos sólidos orgánicos:

- El ingreso de venta del producto final de compostaje y/o lombricultivo (IV): Se calcula por la cantidad de residuos orgánicos generados por cada provincia menos las pérdidas en los procesos de compostaje y/o lombricultivo; obteniendo las toneladas de producto final de cada uno, donde se aplica el precio de venta actual con el crecimiento porcentual para cada año.

- Costo de inversión para planta de compostaje y/o lombricultivo (CTMaq + CTÁrea): Se determina con base a dos subdivisiones de costos. El primero es costo de maquinaria de compostaje y/o lombricultivo y el segundo es costo de área de terreno para la construcción de planta de compostaje y/o lombricultivo. Para el cálculo del primer costo se tienen en cuenta tres grupos de maquinaria con su respetivo número de referencia, que se establece dependiendo de las capacidades técnicas de la máquina para el procesamiento de los residuos sólidos orgánicos generados por cada provincia. El segundo es el cálculo de costo de área de terreno, donde se aplican restricciones al modelo derivadas de la observación a visita de plantas de compostaje y lombricultivo. Las áreas de ocupación de la maquinaria son determinadas con las dimensiones de ancho y largo de la maquinaria.

- El costo de cobro por disposición final de Relleno Sanitario Nuevo Mondoñedo (RSNM) a cada provincia (CRSNM): se calcula mediante el costo actual de tonelada dispuesta que cobra la concesión Relleno Sanitario Nuevo Mondoñedo a cada provincia con Modelo de optimización para planta de compostaje.

\section{Modelo de optimización para el} aprovechamiento en compostaje

Se tiene en cuenta los ingresos de venta de compostaje - (costos totales de maquinaria para compostaje + costos totales de áreas de terrenos para compostaje) - costo de cobro por disposición final de residuos que hace el Relleno Sanitario Nuevo Mondoñedo. Se presenta en la (Ecuación 2).

\section{Ecuación 2. Modelo de optimización para planta de compostaje (MOPC)}

$$
\mathrm{MOPC}=\sum(I V C)-[(\text { CTMaq }+ \text { CTArea })-\text { CRSNM }]
$$

Donde:

IVC = ingreso de venta de compostaje (COLP); CTMaq = costo total de la maquinaria (COLP);

CTÁrea = costo total del área (COLP);

CRSNM = costo del relleno sanitario nuevo Mondoñedo (COLP).

Modelo de optimización para planta de lombricultivo

El modelo de optimización para el aprovechamiento en lombricultivo, se basa en la sumatoria de: ingresos de venta de 
lombricompuestos - (costos totales de maquinaria para lombricultivo + costos totales de áreas de terrenos para lombricultivo) - costos de cobro por disposición final de residuos que hace el Relleno Sanitario Nuevo Mondoñedo. (Ecuación 3)

\section{Ecuación 3. Modelo de optimización para planta de lombricultivo (MOPL)}

$$
\text { MOPL }=\sum(\text { IVL })-[(\text { CTMaq }+ \text { CTArea })-C R S N M]
$$

Donde:

$I V L=$ ingreso de venta lombricompuesto (COLP);

CTMaq = costo total de la maquinaria (COLP);

CTÁrea $=$ costo total del área (COLP);

CRSNM = costo del relleno sanitario nuevo Mondoñedo (COLP).

\section{Discusión}

Las acciones antrópicas son la principal consecuencia de la generación de residuos, causando problemas ambientales, sociales y económicos, por falta de planeación en la construcción de plantas de aprovechamiento óptimas para el procesamiento de residuos sólidos orgánicos ${ }^{6}$. El resultado del compostaje y lombricultivo beneficia en la mejora de suelos, aumentado la absorción y almacenamiento de carbono orgánico dentro los vegetales ${ }^{20}$. La disponibilidad de nitrógeno en el suelo, inactiva plaguicidas e inhibe el crecimiento de patógenos, principales causantes de enfermedades en los seres humano, la aplicación de compost y de lombricompuesto mejora la estructura y la humedad del suelo; por consiguiente, reduce el consumo de fertilizantes químicos que afectan al ambiente 21 .

En Colombia el aprovechamiento de residuos sólidos orgánicos se ve como un proceso aislado dentro del servicio público de aseo. Es por ello, que la mayoría de plantas de aprovechamiento de residuos sólidos orgánicos no cuentan con las condiciones necesarias para obtener la licencia ambiental. El aprovechamiento biológico mediante las técnicas de compostaje y lombricultivo, plantea soluciones óptimas en los enfoques ambientales y económicos. Si bien es cierto, estas técnicas de aprovechamiento biológico contribuyen al uso eficiente energía renovable y modelos de desarrollo limpio (MDL), se debe hacer hincapié en aspectos claves del desarrollo sostenible, como la selección de técnicas y tecnologías apropiadas para las plantas de aprovechamiento de residuos sólidos orgánicos para el departamento de Cundinamarca.

Se tomaron dos referentes para el estado del arte del estudio, el primer estudio es un modelo en programación lineal, donde se estudiaron 32 variables y el objetivo fue minimizar el costo total de funcionamiento y de capital; se elaboró un enfoque en el costo total de tratamiento de residuos y costo de transporte que incluyó las emisiones de $\mathrm{CO}_{2}$, concluyendo que el envío a la instalación de compostaje reduce los $\operatorname{costos}^{21}$. El segundo estudio, se desarrolló en la industria vinícola; se estudió el compostaje desde el enfoque ambiental, técnico y económico como tecnología para reciclar residuos orgánicos que estuvieron siendo incinerados y dispuestos en el vertedero. Se implementó un sistema de compostaje en hileras a gran escala donde utilizaron la composta como sustituto de los fertilizantes; la inversión en maquinaria fue la adquisición de tractor y trituradora para el proceso de compostaje in situ además de parámetro técnicos de dimensiones de pila de compostaje ${ }^{22}$. En contraste al modelo de la industria vinícola, que solo aborda los costos de inversión y de retorno, nuestro estudio incluye parámetros de ingreso por venta de abono orgánico, costo de inversión y costos de disposición, por ende, el modelo integra parámetros eficientes para sostenibilidad en el tiempo de la planta y la reducción de gases efecto invernadero ${ }^{22}$, ya que tiene en cuenta las emisiones de $\mathrm{CO}_{2}$ para cada una de las provincias del Departamento, que fueron calculadas mediante la herramienta de huella de carbono de la gobernación de Cundinamarca ${ }^{16}$.

Fue necesario elaborar un estudio de mercado para obtener información de la maquinaria que comprende el proceso de compostaje y lombricultivo, que permitió proponer un modelo de aprovechamiento conceptual, matemático y 
técnico que responde a soluciones de enfoque ambiental, económicas y sociales; aporta a la sustentabilidad en sus tres enfoques: el primero mediante el ahorro de emisiones de efecto invernadero, esto se puede explicar porque al mejorar la fertilidad en suelos, se transforma y captura $\mathrm{CO}_{2}$, disminuyendo la emisión de gases en la degradación de los residuos orgánicos y evitando la contaminación de aguas por generación de lixiviados. El segundo enfoque percibe ingresos por la venta del compost o lombricompuesto, además mejora la economía local. En el tercer enfoque, de carácter social, se reduce la construcción de rellenos sanitarios gracias al aprovechamiento de los RSO, se disminuye el impacto en zonas vulnerables y genera beneficios en el incremento de empleo formal, promoviendo la "consciencia ambiental" en la comunidad dónde se desarrolla el proyecto de la planta de aprovechamiento.

Existen vacíos científicos en modelos de aprovechamiento de residuos sólidos orgánicos porque no incluyen el cálculo de ahorro de emisiones de $\mathrm{CO}_{2}$ y al mismo tiempo parámetros técnicos para optimización del proceso de compostaje y/o lombricultivo ${ }^{22}$; por esto, el modelo sugerido involucró los enfoques ambiental, económico y social, para responder al globo de sustentabilidad.

La acumulación de los residuos sin ningún tipo de aprovechamiento amenaza directamente al ambiente concentrando contaminantes que impacta directamente la salud humana. Para la gestión sostenible de los residuos sólidos orgánicos, es necesario generar estrategias de planificación, por ello, con la optimización del aprovechamiento de compostaje y/o lombricultivo se ampliará el suministro de energía renovable, puesto que las técnicas de tratamiento biológico respondieron a un número de necesidades ambientales, sociales $y$ económicas.

La fracción orgánica de residuos es responsable de emisiones del 5\% total de los gases efecto invernadero en el mundo ${ }^{21}$. Los residuos sólidos orgánicos son ricos en carbono y nitrógeno, la adición de compost y lombricompuesto favorecen la incorpocación de carbono al suelo, y su liberación gradual junto con nitrógeno. El compost mitiga los impactos del GEI (Gas Efecto Invernadero), porque permite el crecimiento de plantas, gracias al incremento de la absorción y fijación del carbono, hecho que resalta la importancia del proceso de compostaje como mecanismo de desarrollo limpio para la reducción de GEI y el desarrollo sostenible para los países ${ }^{15}$. Los beneficios que ofrece el lombricompuesto son: el incremento en la eficiencia de fertilización, la inactivación de residuos de plaguicidas, el control de poblaciones de hongos y bacterias patógenas y el mejoramiento de la humedad, permeabilidad y aireación del suelo ${ }^{22}$.

El enfoque del modelo de este estudio presenta un esquema de aprovechamiento biológico para el compostaje aeróbico y lombricultivo, que busca la optimización de los procesos ambientales, técnicos y económicos para la construcción de plantas de aprovechamiento de residuos sólidos orgánicos para el departamento de Cundinamarca. Por ello se desarrollaron ecuaciones de optimización para definir ingresos de producto final, costos de inversión y costos de recaudo por disposición final en relleno. En la aplicación del modelo de aprovechamiento, se estableció la viabilidad para la construcción de plantas de aprovechamiento de residuos sólidos orgánicos a través del cálculo de las utilidades, partiendo de la base económica de utilidad en el intervalo 2010 a 2040, para cada una de las provincias del departamento de Cundinamarca. El modelo permite sugerir el número de plantas que son posibles construir por provincia.

Se estableció el cálculo de las variaciones de emisiones en toneladas equivalentes $/ \mathrm{CO}_{2}$, con la implementación de las técnicas de aprovechamiento biológico como compostaje y lombricultivo. Las emisiones de ahorro que se presentan en el capítulo de resultados corresponden a toneladas equivalentes $/ \mathrm{CO}_{2}$ que se dejan de emitir al ambiente, mediante el reciclaje de la fracción orgánica para evitar costos ambientales y económicos. 


\section{Conclusiones}

El modelo de optimización para las plantas de compostaje y lombricultivo del departamento de Cundinamarca, se desarrolla bajo criterios técnicos, matemáticos, ambientales y económicos propios de la investigación. Es por ello, que la ecuación de optimización parte de la base económica de utilidad con el fin de determinar la sostenibilidad de las plantas en el tiempo, teniendo en cuenta los ingresos de venta del producto final, costos de inversión, y la reducción del costo en su totalidad por la disposición final de los residuos sólidos orgánicos en el relleno.

El modelo para plantas de compostaje y lombricultivo es una herramienta que apoya a los tomadores de decisión a definir la viabilidad técnica, económica y ambiental en la construcción de plantas de aprovechamiento de compostaje y/o lombricultivo.

El aprovechamiento de RSO permite recudir las emisiones de toneladas en $\mathrm{CO}_{2}$ emitidos a la atmósfera. Los productos finales del compostaje y lombricultivo reducen las emisiones de gases efecto invernadero capturando el $\mathrm{CO}_{2}$ en abonos y fertilizantes orgánicos, de modo que el modelo es ambientalmente sostenible en el tiempo. Es importante establecer que las estrategias de separación en la fuente de los residuos sólidos orgánicos, evitando que el proceso de compostaje y lombricultivo se encuentre contaminado por la presencia de metales pesados y/o de patógenos, con ello se asegura que los abonos y fertilizantes orgánicos cumplan con estándares de calidad beneficiando al ambiente.

\section{Agradecimientos}

Los autores agradecen de manera especial a la Gobernación Departamento de Cundinamarca y a la Universidad Distrital Francisco José de Caldas, por el apoyo para el desarrollo de la investigación.

Conflicto de intereses: Ninguno declarado.

\section{Referencias}

1. Gardner G. Municipal Solid Waste Growing. Worldwatch Vital Signs. 2012 April. Disponible en: http://vitalsigns.worldwatch.org/vs-trend/ municipalsolid-waste-growing.(2nd).

2. Pires A, Martinho, Chang NB. Solid waste management in European countries: A review of systems analysis techniques. Journal of Environmental Management. 2011 December; $92(1033$-1050).

3. Wen-cong L, Yong-xi M, Bergmann H. Technological Options to Ameliorate Waste Treatment of Intensive Pig Production in China: An Analysis Based on BioEconomic Model. Journal of Integrative Agriculture. 2014 February; 13(443-454).

4. Juul N, Münster M, Ravn H, Söderman M. Challenges when performing economic optimization of waste treatment: A review. Waste Management. 2013 September; 33(1918 - 1925).

5. Ahmed Bazmia A, Zahedia G. Sustainable energy systems: Role of optimization modeling techniques in power generation and supply-A review. Renewable and Sustainable Energy Reviews. Renewable and Sustainable Energy Reviews. 2011 October; 15(3480 3500).

6. SSPD. Diagnóstico Sectorial de Plantas de Aprovechamiento de Residuos Sólidos. Sectorial. Bogotá: Documento de Superintendencia de Servicios Públicos Domiciliarios, Colombia; 2008. Report No.: http://www.superservicios.gov.co/

7. Abu Qdais H, Abdulla F, Qrenawi L. Solid Waste Landfills as a Source of Green Energy: Case Study of Al Akeeder Landfill. Jordan Journal of Mechanical and Industrial Engineering. 2010 Jun; 4(69-74).

8. Lino F, Bizzo W, Da Silva E, Ismail K. Energy impact of waste recyclable in a Brazilian metropolitan. Resources, Conservation and Recycling. 2010 September; 54(916-922).

9. Lino F. Consumo de Energia no Transporte da Coleta Seletiva de Residuo Sólido Domiciliar no Município de Campinas (SP). Universidade Estadual de Campinas. 2009 May; FEM/UNICAMP. Campinas, Brasil.(s.n.).

10. MAVDT. Construcción de criterios técnicos para el aprovechamiento y valorización de residuos sólidos orgánicos con alta tasa de biodegradación, plásticos, vidrio, papel y cartón. MAVDT. 2008 Diciembre; Manual: Generalidades 1(25 - 91.).

11. Gobernación Cundinamarca. PGIRS. Plan de gestión integral de residuos orgánicos de Cundinamarca. 2014.

12. FAO. Estimación de emisiones de gases efecto invernadero en la agricultura. Manual par abordar los requisitos de los datos para los paises en desarollo. Roma: Organización de las naciones unidas para la alimentación y la agricultura; 2015.

13. Vergel. Metodología. Un manual para la elaboración de diseños y proyectos de investigación. Compilación y ampliación temática. Publicaciones Corporación UNICOSTA. Barranquilla. 2010.

14. Hurtado J. Metodología de la investigación holística. Direccción de Investigaciones y Postgrado ed. UNA, 
editor. Caracas - Venezuela: Universidad Nacional Abierta; 2000.

15. Minoglou M, Komilis D. Optimizing the treatment and disposal of municipal solid wastesusing mathematical programming-A case study in a Greek region. Conservation and Recycling. 2013 November; 80(46 57).

16. Gobernación de Cundinamarca. Huella de carbono CO2. Disponible en:

http://huellacarbono.cundinamarca.gov.co/modules/ mod_calculo_personal/

17. Rogger C, Beaurain F, \& S. Sc. Composting projects under the Clean Development Mechanism: Sustainable contribution to mitigate climate change. Waste Management. 2011.

18. Gobernación de Cundinamarca. Huella de carbono CO2. 2014. Disponible http://huellacarbono.cundinamarca.gov.co/modules/ mod_calculo_personal/

19. Rincón Soto C. Guía de costos para micro y pequeños empresarios: una manera fácil y sencilla de creer Bogotá D.C: ECOE EDICIONES; 2011.

20. Púlido J. Entrevista en residuos sólidos orgánicos (S. Castañeda E, editor.; 2014.

21. Miller C, Heeren V, Hornsby J. Matemática y Razonamiento y Aplicaciones. Décima Edición ed. México: Pearson; 2006.

22. Ruggieri L, Cadena E, Martinez-Blanco J, y otros. Recovery of organic wastes in the Spanish wine industry. Technical, economic and environmental analyses of the composting process. Journal Cleaner Production. 2009 June; 17(830-838) 